\title{
Médiévales
}

Langues, Textes, Histoire

53 | automne 2007

La nature en partage

\section{Miracle et apologétique : sur quelques exempla anti- hussites dans le Formicarius de Jean Nider}

Miracle and apologetics : four

Olivier Marin

\section{OpenEdition}

1 Journals

Édition électronique

URL : https://journals.openedition.org/medievales/4022

DOI : $10.4000 /$ medievales. 4022

ISSN : 1777-5892

Éditeur

Presses universitaires de Vincennes

Édition imprimée

Date de publication : 1 décembre 2007

Pagination : 143-164

ISBN : 978-2-84292-211-5

ISSN : 0751-2708

Référence électronique

Olivier Marin, « Miracle et apologétique : sur quelques exempla anti-hussites dans le Formicarius de Jean Nider ", Médiévales [En ligne], 53 | automne 2007, mis en ligne le 17 décembre 2009, consulté le 23 avril 2022. URL : http://journals.openedition.org/medievales/4022 ; DOI : https://doi.org/10.4000/ medievales.4022

Tous droits réservés 
Olivier MARIN

\section{MIRACLE ET APOLOGÉTIQUE : SUR QUELQUES EXEMPLA ANTI-HUSSITES DANS LE FORMICARIUS DE JEAN NIDER*}

Si le miracle est une donnée permanente et universelle dans l'histoire du christianisme médiéval, ses usages ont varié au gré des intérêts changeants qu'en attendaient l'Église et ses porte-parole. Les travaux d'A. Vauchez, notamment, ont montré qu'à partir de la fin du XII ${ }^{\mathrm{e}}$ siècle, la menace cathare avait incité les hagiographes à enrôler la sainteté et ses manifestations miraculeuses au service de la défense de la vraie foi ${ }^{1}$. Cette étude voudrait éclairer comment la conjonction entre miracle et apologétique s'est recomposée dans les années 1420-1430, à une époque où l'Église était confrontée en Bohême à un défi hérétique d'une ampleur telle qu'elle n'en avait pas connu depuis deux siècles. Or dans ce contexte, les formules éprouvées ne suffisaient plus. Malgré son dénouement heureux, le Grand Schisme avait laissé des traces : en brouillant les frontières de l'Église, il avait rendu moins naturel le lien du salut avec l'appartenance à l'institution. Le succès de la dissidence hussite au cœur de l'Europe, puis le conflit recommencé entre le pape et le concile n'avaient fait qu'approfondir la difficulté qu'éprouvaient fidèles et théologiens à reconnaître la véritable Église. C'en était fini d'une certaine idée de la Chrétienté et, avec elle, des médiations qui assuraient l'accès du visible à l'invisible ${ }^{2}$.

* Une première version de ce travail a été présentée en tchèque au Centre des Études Médiévales (Centrum medievistických studií), à Prague, le 21 avril 2006. Que tous les participants, notamment D. Foltýn, M. Nejedlý, M. Nodl, P. Sommer et F. Šmahel, soient remerciés pour leurs remarques et suggestions, dont j'ai essayé de tenir compte.

1. A. VAuchez, Saints, prophètes et visionnaires. Le pouvoir surnaturel au Moyen Âge, Paris, 1999, en particulier le chapitre 12 «a sainteté, arme contre l'hérésie : la Vie de Marie d'Oignies par Jacques de Vitry », p. 175-188.

2. Sur cette question, voir l'article classique de S. H. HendRIX, «In Quest of the Vera Ecclesia. The Crise of Late Medieval Ecclesiology », Viator 7, 1976, p. 347-378. 
Jean Nider (vers 1380-1438) fut de ceux qui tentèrent de répondre à la crise. Ce dominicain souabe s'était trouvé aux premières loges pour assister, d'abord comme maître en théologie à l'université de Vienne, puis au couvent de Bâle dont il était devenu le prieur, aux grands débats ecclésiologiques et pastoraux de son temps. Une telle expérience personnelle fait la valeur historique du grand œuvre qu'il acheva en 1437, une fois revenu à Vienne, et qui est passé à la postérité comme une des sources du Marteau des Sorcières, le Formicarius. Il s'en faut pourtant de beaucoup que l'ouvrage traite seulement de sorcellerie. Depuis une demi-dizaine d'années, les travaux de W. Tschacher et de M. D. Bailey, en attendant l'édition critique préparée par C. Chène (université de Lausanne), ont permis de lui rendre sa mesure ${ }^{3}$. Sous la forme d'un dialogue entre un théologien et son étudiant, curieux mais paresseux, la «Fourmilière » passe en revue les mœurs des fourmis et les interprète allégoriquement comme l'image des actions extraordinaires, bonnes ou mauvaises, dont sont capables les chrétiens; des histoires contemporaines édifiantes viennent illustrer chaque chapitre. Chemin faisant, Nider nous offre un véritable miroir de son siècle, qui allie une documentation exceptionnellement vivante et un propos ambitieux : montrer aux chrétiens d'Allemagne désemparés, qui doutent de la Providence comme jadis les Hébreux dans le désert, que Dieu continue, envers et contre tout, à accomplir des merveilles à travers l'histoire ${ }^{4}$. L'entreprise n'était pas sans danger, et Nider le sait. La confusion des signes guette. Aussi met-il un point d'honneur à débrouiller le vrai du faux, à distinguer le saint du possédé, à débusquer l'hérétique sous les apparences du fidèle. Le plan général reflète cet immense travail de discernement des esprits qui, depuis quelques décennies, mobilisait les théologiens soucieux de direction spirituelle ${ }^{5}$ : aux livres I, II et IV, qui traitent «Des exemples et des œuvres exceptionnelles des hommes de bien», «Des bonnes révélations vraisemblables» et «Des œuvres vertueuses de

3. W. Tschacher, Der Formicarius des Johannes Nider von 1437. Studien zu den Anfängen der europäischen Hexenverfolgung im Spätmittelalter, Aix-la-Chapelle, 2000 ; M. D. BAILEY, Battling Demons. Witchcraft, Heresy and Reform in the Late Middle Âges, University Park, Pennsylvania, 2002. Il existe déjà une traduction française commentée du livre 5 du Formicarius par J. Ceard, D. Teysseire, N. Jacques-Lefèvre et S. Houdard, qui a paru sous le titre Jean Nider, Des sorciers et leurs tromperies, Grenoble, 2005.

4. Ainsi qu'il le déclare dans le prologue : «Peragrans crebro partes quasdam presertim Alemanie querelas nonnumquam audivi pigrorum in fide hominum : Cur modo inter Christicolas Deus nullis ecclesiam roboret miraculis, aut sacris pro fide tuenda et virtutibus ad bene vivendum non illuminet, sicut olim, revelationibus ? Cum perfidis enim Iudeis clamitant : Signa nostra non vidimus, iam non est propheta et nos non cognoscet amplius (Ps. 73, 9). Quod murmur, Christi cooperante gratia, compescere conabor in sequentibus per plura que prope et circa nostra tempora, imo nobis dum datum est vivere in humanis, mira a Deo vel miracula ostensa sunt divinitus, cum revelationibus variis et sanctorum hominum virtutibus... » (page non numérotée). Nous avons utilisé l'édition de Douai, parue en 1602 chez G. Colvener.

5. Sur cette dimension du Formicarius, voir G. KLANICZAY, « Entre visions angéliques et transes chamaniques : le sabbat des sorcières dans le Formicarius de Jean Nider », Médiévales, 44, 2003, p. 47-72. 
ceux qui ont atteint la perfection », font pendant les livres III et V, dont le but est de décrire successivement « les visions fausses et illusoires » et «les sorciers et leurs erreurs fallacieuses ».

Or l'interrogation centrale du Formicarius, celle de la duplicité des signes providentiels, se posait avec une acuité particulière quand on se remémorait en 1437 les quelque quinze années de confrontation armée avec l'hérésie bohémienne. L'échec des cinq croisades promulguées contre les sectateurs de Jean Hus n'avait-il pas couvert de honte les armées catholiques ? Nulle assistance n'était alors venue d'en-haut. Non seulement Dieu avait permis que les hérétiques triomphent des assauts portés contre eux et ravagent les pays avoisinants, mais il avait fallu que l'Eglise, chose inouie, accepte par la voix du concile de traiter avec eux. Beaucoup parmi les chroniqueurs du temps s'étaient émus de ce scandale pour la foi. Comme eux, Nider se plaint que les soldats catholiques ont tourné les talons dès les premières escarmouches et voit dans leurs défaites un avertissement divin ${ }^{6}$. Mais ce point de vue moralisateur ne lui suffit pas. En théologien qu'il est, il se montre aussi capable de livrer un exposé circonstancié, d'ordre à la fois historique et doctrinal, de l'hérésie tchèque, qui occupe les chapitres 9,10 et $12 \mathrm{du}$ troisième livre. C. Chène vient d'en résumer l'argument et d'en souligner la charge polémique, en accord avec le projet global de stigmatisation des visions diaboliques que s'est assigné l'auteur du Formicarius ${ }^{7}$.

Pour autant, le douzième et dernier chapitre ne se laisse pas réduire aux clichés de la controverse anti-hussite. En guise d'introduction, Nider met dans la bouche du Paresseux une interrogation inquiète, qui fait directement écho au prologue : "Je désire savoir si d'aventure en ce temps-là il est arrivé parmi les fidèles certains miracles propres à raffermir la foi. Dieu, rétorque le Théologien, n'a pas cessé jusqu'à maintenant de faire jaillir la fontaine de sa miséricorde sur de nombreux fidèles, qui attestent avoir reçu cette aide par grâce ${ }^{8}$. » Et de citer à l'appui quatre récits exemplaires censés prouver que, malgré les innombrables avanies subies, Dieu n'a pas abandonné son peuple. Ces exempla n'ont pas encore retenu l'attention qu'ils méritent. Il s'agira ici de montrer comment ils font jouer un ressort puissant, encore qu'il soit souvent méconnu des historiens, de la résistance catholique au hussitisme. Car si l'on connaît bien aujourd'hui les moyens diplomatiques, militaires et intellectuels mis en œuvre par l'Église pour endiguer la contestation

6. On pourra comparer sur ce point l'attitude de Nider avec celle d'André de Ratisbonne étudiée par N. Housley, «Explaining Defeat: Andrew of Regensburg and the Hussite Crusades », dans M. Balard, B. Z. KeDAR et J. RILEY-Smith (dir.), Dei gesta per Francos. Études sur les croisades dédiées à Jean Richard, Londres, 2001, p. 87-95.

7. C. CHÊNE, "L'hérésie hussite vue par un dominicain observant: le Formicarius de Jean Nider (ca. 1380-1438) », dans A. De LANGE et K. Utz Tremp (dir.), Friedrich Reiser und die 'waldensisch-hussitische Internationale' im 15. Jahrhundert, Heidelberg, 2006, p. 317-340.

8. «Piger : Cupio scire, anne eodem tempore aliqua miracula inter fideles pro fidei roboratione facta sint alicubi ? Theologus : Non destitit Deus fontem sue misericordie protunc emanare super multos fideles, quibus gratiose supervenisse constat » (p. 254). 
bohémienne, il n'en va pas de même du recours, pourtant déterminant dans les mentalités du temps, au miraculeux. Quels événements Jean Nider a-til choisis et pourquoi les a-t-il jugés dignes de recevoir une interprétation providentialiste? Avant de s'arrêter au contenu de ces quatre exempla, il conviendra de comprendre quelles étaient ses conditions de travail et, par là, les outils intellectuels dont il disposait pour contrecarrer le hussitisme. Puis l'interprétation sera menée selon les règles qui gouvernaient l'allégorie médiévale : seule l'élucidation préalable de la littera, autrement dit des faits et de leurs circonstances, permettra d'en dégager les possibles sens figurés (sensus).

TRADUCTION (Formicarius III, 12)

« Nous revenions de la cité d'Eger, aux frontières de la Bohême, où de la part du concile de Bâle, nous avions promis aux hérétiques de leur accorder une audience s'ils s'y présentaient ; comme nous étions parvenus avec le seigneur Frédéric, margrave du Brandebourg, à l'une de ses villes fortes appelée Culmach, au-dessus de laquelle un promontoire porte un château inexpugnable du nom de Plassemberg, le margrave nous raconta une merveille qui s'y était produite peu de temps avant. Les hérétiques avaient en effet pénétré dans la région avec une si grande force que personne ne pouvait y résister et qu'ils ne tardèrent pas à s'emparer de la place. Moines, clercs et moniales, Juifs et habitants fuirent donc en grand nombre aussi bien les localités voisines que la ville forte elle-même pour chercher asile dans ce château. Cependant, les hommes d'armes qui servaient dans le château faisaient de temps à autre des sorties avec leurs balistes et leurs bombardes à main : ils descendaient la pente surplombant la place et lançaient autant de projectiles qu'ils pouvaient contre les mécréants, tout en attendant la riposte de ces hérétiques. Or un jour que les nôtres descendaient du château selon leur pratique habituelle, ils prirent avec eux un Juif qui était tout à fait rompu à la technique des bombardes à main. À leur vue, les hérétiques coururent aussitôt à l'église de la ville, en sortirent une statue du Christ au Mont des Oliviers qui était faite en pierre ou en brique et la portèrent sur la place de manière à faire voir aux nôtres l'outrage qu'ils lui réservaient. Comme les nôtres regardaient ce que ces perfides leur présentaient d'incongru, ils virent un mécréant s'approcher de la statue figurant le Sauveur en prière les mains levées, tirer son épée et s'exclamer d'une voix forte et moqueuse : "Voici votre Dieu, s'il le veut, qu'il se libère lui-même !». Et il décapita la statue d'un coup. Devant ce spectacle, le Juif prépara sa bombarde au tir et déclara aux chrétiens : "Quoique je sois d'une autre foi que vous, je défendrai la vôtre de tout mon possible. » Il mit donc le feu à la machine et, du premier coup, toucha à mort l'hérétique coupable, à l'émerveillement général. 
Un autre jour arriva un autre prodige. Il y avait dans le château un homme idiot depuis la naissance, très impétueux et bavard, qui chaque jour désirait sortir du château avec les hommes d'armes et menaçait d'engager seul le combat contre les mécréants hérétiques ; c'est pourquoi on le surveillait étroitement pour l'empêcher de sortir du château. Mais un jour que le garde s'était éloigné de la porte, il saisit l'occasion et trouva le moyen, soit à l'aide de clés soit autrement, de sortir à l'insu de tous. Il descendit donc la pente, saisit un bâton, se mit même à courir et accabla de paroles brutales et de coups les hérétiques qu'il trouvait à proximité de la place. Ceux-ci tirèrent leurs épées pour se défendre et rouèrent l'idiot de blessures très profondes (j'en vis les cicatrices par la suite) et, pensaient-ils, fatales. Le petit pauvre du Christ s'effondra donc et resta gisant dans son sang longtemps après que les hérétiques l'eurent abandonné. Mais les forces lui revinrent finalement, il se releva et entreprit de remonter au château, à la stupéfaction des gardes qui, sur les remparts, se demandaient comment et quand l'idiot avait bien pu s'échapper. Lui ouvrant donc, ils le trouvèrent couvert de blessures mortelles. Car il apparut qu'entre autres endroits, il avait été poignardé à la poitrine, une localisation qui d'habitude, étant donné la profondeur de la blessure, entraine toujours la mort. Or l'idiot avait toujours eu en sainte horreur les Juifs, et les hérétiques davantage encore ; et alors qu'on lui amenait pour le soigner un Juif fort expert en chirurgie, il le repoussa comme s'il avait vu la mort. «Loin de moi, dit-il, de me laisser soigner par un hérétique. » Il se précipita affamé vers le cuisinier dans sa cuisine, avala une bouchée et en l'espace de quelques jours, sans opération ni intervention médicale, il était guéri. Tous jugèrent qu'il n'avait pu l'être que par le secours divin, puisqu'il n'avait quasiment pas bénéficié de l'assistance humaine.

Il était une fois à Prague des ambassadeurs du concile de Bâle : l'évêque de Coutances, de la province de Rouen, l'évêque d'Augsbourg, l'archidiacre de Barcelone, le doyen de Tours et de nombreux docteurs en droit canon et civil s'employaient, durant les trêves qu'ils avaient obtenues, à ramener les mécréants dans le sein de l'Église ; ils étaient accompagnés d'une nombreuse suite, mais tous observaient un appareil simple et digne de leur état ecclésiastique. Or leur séjour traînait de longs mois et semaines à cause de l'obstination des mécréants, et voici qu'une pestilence très violente se déchaîna dans le peuple, tuant chaque jour à peu près cent personnes. C'est alors qu'à l'étonnement général des mécréants, Dieu fit merveille dans le logis des nôtres : alors que la pestilence sévissait fortement dans toutes les habitations voisines ou plus lointaines, elle ne pénétra jamais dans leur maison, de sorte qu'au cours de leur séjour, aucun fidèle ne succomba ni ne fut même atteint de la peste.

Je ne parlerai pas (pour ne pas encourir le reproche de complaisance) du sous-prieur du couvent de Franrenstein (sic) qui, s'étant édifié dans notre ordre, demeura jusqu'au bout solidement et ardemment fidèle à la règle : quand les hérétiques le firent prisonnier dans son couvent, il s'en tint avec la plus grande fermeté à la foi catholique et, dans sa patience, il supporta le 
bûcher avec une telle équanimité que les mécréants avouèrent n'en avoir jamais trouvé de pareille parmi les nôtres face à la mort.

$$
* *
$$

\section{Nider au travail}

Ces exempla ne peuvent se comprendre qu'à la lumière des conditions concrètes dans lesquelles le dominicain a composé son ouvre et recueilli ses informations. Son point de vue sur le hussitisme n'est pas celui de Sirius : il se révèle tributaire d'une expérience autobiographique, de réseaux de relations et de modèles d'écriture qu'il faut identifier.

Quand Jean Nider mit en forme ces quatre récits, cela faisait plus d'une décennie que le hussitisme lui était devenu une réalité familière. Dès le début des années 1420, quand ses études l'avaient mené à l'université de Vienne, le jeune théologien avait vu comment les hérétiques enflammaient la Bohême voisine et menaçaient à l'occasion les frontières autrichiennes. Il eut bientôt à payer lui-même de sa personne. Le Formicarius nous apprend qu'à une date inconnue (1427 ?), il prêcha la croisade contre les Bohémiens ${ }^{9}$. Puis il prit la plume au printemps 1430 pour réfuter leurs thèses. L'œuvre, intitulée Tractatus contra heresim Hussitarum, n'est plus connue que par deux manuscrits incomplets et ne semble pas avoir dépassé une diffusion confidentielle. Mais Nider avait ainsi acquis une connaissance assez intime du hussitisme pour que le concile de Bâle le désignât le 28 novembre 1431 parmi ses représentants chargés de négocier à Nuremberg une trêve avec les Hussites. Il fut même au printemps suivant l'un des principaux artisans de l'accord conclu à Cheb (Eger), qui fixait les règles herméneutiques du dialogue futur et ouvrait la voie à une participation des Bohémiens au concile ${ }^{10}$.

Une fois revenu à Bâle en juin 1432, notre dominicain ne participa certes plus directement aux pourparlers, mais la fin du troisième livre du Formicarius montre quel intérêt il conserva pour les affaires tchèques. L'étude récente de $\mathrm{C}$. Chêne nous dispense d'entrer dans les détails. Retenons qu'au fil de ses explications doctrinales, le Théologien insiste en particulier sur la question de l'autorité de la Bible et de la tradition qui avait été si longuement débattue à Cheb. Il évite en revanche de traiter de la communion sous les deux espèces, conscient que cet usage est hors de cause depuis que les Compactata l'ont formellement autorisé en Bohême. Seuls les excès des radicaux en matière de discipline sacramentelle sont donc fustigés ${ }^{11}$. Au total,

9. «Piger : Quia te scio saepe tempore quo expeditio contra eos fieri debuit, et crux predicari per Theutoniam, eandem contra eos crucem predicasti » (p. 237-238).

10. Bonne mise au point sur le sujet dans M. D. BAILey, Battling Demons..., op. cit., p. $57-64$.

11. Livre 3, chapitres 9 et 10 , p. 237-247. 
Nider se montre un excellent observateur des rapports de force en Bohême. Il perçoit bien les dissensions au sein du camp hussite, divisé entre Taborites, Orphelins et Pragois, lesquels se partageaient à leur tour selon leur affiliation à la Vieille ou à la Nouvelle Ville. Et alors que tant de ses contemporains se réjouissaient un peu vite de la «réduction» des hérétiques tchèques à la suite de leur défaite à Lipany, lui sait que la partie n'est pas gagnée tant que les Taborites ne sont pas tous écrasés ${ }^{12}$. On le voit, Nider n'est pas dans ce domaine un naï avaleur de rumeurs. Sa longue fréquentation des textes comme des hommes de Bohême a aiguisé son esprit critique et orienté son écoute des hussitica.

Avec le douzième chapitre du troisième livre, le lecteur passe à des événements quasi contemporains du Formicarius et dont la plupart n'ont pas dépassé l'échelle locale. D'où Nider tient-il au juste ses informations ? Le dominicain écrit dans le prologue s'être fondé sur ce qu'il a vu et entendu personnellement ou sur les témoignagnes de personnes dignes de foi ${ }^{13}$. Aucun des quatre exempla susdits n'entre dans la première catégorie : Nider n'a pas été le témoin oculaire de ce qu'il rapporte ici. En revanche, il nous informe que les deux premiers récits lui ont été racontés par le margrave de Brandebourg Frédéric lorsque, de retour de Cheb, ils firent halte ensemble dans le château de Plassemburg sur Kulmbach ${ }^{14}$. De fait, le prince électeur avait participé en sa qualité de premier chancelier de l'Empire aux négociations avec les Pragois et, à la fin du mois de mai 1432, il accompagna dans sa résidence franconienne les membres de la délégation conciliaire qui s'en revenaient à Bâle. Nider eut alors tout loisir de s'entretenir avec ce protagoniste de premier plan. Il put de surcroît vérifier ses dires en recueillant sur place le témoignage du simple d'esprit qui apparaît dans le deuxième exemplum et dont il vit ensuite, note-t-il, les cicatrices ${ }^{15}$. Comme souvent, l'auteur du Formicarius entendait là pratiquer une observation directe qui lui permette d'authentifier ce qu'il avance.

On ne peut pas en dire autant des deux derniers exempla, pour lesquels le dominicain ne cite pas la moindre source. Faut-il penser, comme le suggère $\mathrm{W}$. Tschacher, que l'anecdote sur la peste provient de l'archidiacre de Barcelone Jean Palomar, l'un des chefs de file de l'ambassade bâloise à Prague ?

12. «Theologus : Multi sunt in regno praefato non ambigo catholici, sed mihi credas ibi ignis dudum accensus nondum ad plenum extinctus est, ut in Thaboritis ardet, utinam non in multis aliis » (p. 237).

13. «Que constant, aut visus mei, vel auditus propria experientia, aut fide dignorum relatibus et, ut reor, satis exinde facta sunt credibilia » (page non numérotée).

14. «Cum etiam de metis Bohemie a civitate Egressei venissemus, ubi ex parte Basiliensis concilii hereticis audientiam sibi dari, si venirent, promisimus, venimus in comitatu domini Frederici marchionis Brandenburgensis, in oppidum suum quod Culmach dicitur, super quo castrum inexpugnabile in alto jacet in monticulo dictum Plassemberg, ibi mirum tale narrante nobis Marchione paulo ante evenerat » (p. 254). Voir là-dessus F. ŠMAHEL, Die hussitische Revolution, tome 3, Hanovre, 2002, p. 1544.

15. « ... quorum cicatrices vidi postmodum » (p. 256). 
Certes, les deux hommes se connaissaient bien: non seulement ils se côtoyèrent dès les débuts du concile, mais Nider se rendit avec lui en 1434 à la diète de Ratisbonne ${ }^{16}$. Mais c'est oublier que le Tout-Bâle avait eu connaissance de l'événement dès l'hiver précédent. Martin Berruyer le 13 janvier, puis Jean Palomar le 15 février, avaient annoncé au concile comment l'épidémie de peste avait providentiellement épargné leur délégation. La nouvelle fit grand bruit : nombreux sont les témoins à la rapporter dans leur journal ou leur correspondance ${ }^{17}$. À cet égard, le Formicarius ne fait que colporter un récit déjà largement répandu et que Nider suppose connu de ses lecteurs.

Quant à l'exemplum relatif au sous-prieur de Frankenstein, rien ne permet d'en détecter avec certitude l'origine. Il est seulement permis d'imaginer que Nider l'a entendu circuler au sein de son ordre. Comme vicaire du mouvement observant en Allemagne, il était en effet activement engagé dans la réforme de nombreux couvents masculins comme féminins et appartenait au premier cercle du gouvernement des Frères Prêcheurs. Il accompagna ainsi le maitre général Barthélemy Texier dans certains de ses déplacements en $1428^{18}$. Dans ces conditions, Nider disposait de contacts assez étendus pour savoir quelles épreuves s'étaient abattues sur ses confrères de la province voisine de Pologne. Quelque six années avant qu'il ne compose son Formicarius, un autre dominicain, Hermann Korner de Lubeck, en avait déjà recueilli l'écho dans sa Chronica novella; le martyre des frères de Frankenstein y figurait en bonne place, preuve de sa rapide notoriété ${ }^{19}$. Reste que Nider ne puise pas à cette source et que dans ce cas comme dans le précédent, il ne s'est pas rendu sur le lieu du miracle. Connue de deuxième ou de troisième main, son information prête alors bien davantage à caution.

Le travail de Nider ne s'arrêtait du reste pas là. Du fait rapporté, il lui fallait faire un exemple, soigneusement accordé aux leçons morales et reli-

16. Der Formicarius..., op. cit., p. 515.

17. Voir la lettre du bénédictin Ulrich Stoeckel du 19 février 1434, dans Concilium basiliense, éd. J. Haller, t. 1, Bâle, 1896, p. 80, ou encore le journal anonyme, dans Concilium basiliense, éd. G. BeCKMAnN, t. 5, Bâle, 1904, p. 76 et 81. André de Ratisbonne en fit lui aussi mention dans sa continuation de la Chronica pontificum et imperatorum Romanorum (éd. G. LEIDINGER, Andreas von Regensburg, Sämtliche Werke, Munich, 1903, p. 480-481): «Denique ambasiatoribus concilii Prage existentibus non erat domus, in qua non esset mortuus aut infirmus, preterquam in domo ambasiatorum, cunctis dicentibus, quod hec fierent iusto iudicio divine ulcionis ».

18. Cf. W. Tschacher, Der Formicarius..., op. cit., p. 51-60.

19. Dans sa version C, aujourd'hui perdue, mais qui nous est connue par la Chronique dite de Rufus (éditée dans Die Chroniken der niedersächischen Städte - Lübeck, Leipzig, 1902, ici p.308). La version D ultérieure a été éditée par les soins de J. SchwaLm, Die Chronica novella des Hermann Korners, Göttingen, 1895. Sur ces sources et les témoignages qu'elles fournissent au sujet de la situation tchèque, voir G. DiEHL, "'De Romexhe coningh van Behemen' und 'de kettere van Bemen' : Die Wahrnehmung Böhmens in der Lübecker Chronistik des späten Mittelalters », dans D. Fliegler et V. BoK (éd.), Deutsche Literatur des Mittelalters in Böhmen und über Böhmen, Vienne, 2001, p. 319-342. 
gieuses qu'il voulait donner. Il est vrai que sur les deux derniers récits, son intervention pouvait se réduire au minimum, puisque l'un et l'autre avaient déjà été apprêtés à des fins d'édification. Aussi bien les réduit-il à quelques phrases, dont la fonction ne peut guère être que celle d'une abréviation mnémotechnique. Les matériaux collectés par Nider à Kulmbach sont en revanche originaux et donnent l'impression de se rapprocher de l'oralité et de sa fraîcheur. Gardons-nous de croire cependant que ces deux premiers exempla livrent un écho direct de ce que le dominicain a entendu. Il faut d'abord compter avec le filtre de la langue : Nider a traduit en latin ce qui lui fut raconté en allemand. Surtout, il s'est efforcé de les couler dans la forme de l'exemplum, c'est-à-dire, selon la définition classique qu'en a donnée J. Le Goff, d' «un récit bref donné comme véridique et destiné à convaincre l'auditoire par une leçon salutaire ${ }^{20} »$.

Ce faisant, Nider réemployait un moyen rhétorique prisé depuis l'Antiquité et que les prédicateurs, spécialement dominicains, avaient déjà abondamment illustré. À suivre J. Berlioz, il semble que le Formicarius renoue plus précisément avec les grands recueils d'exempla du XIII ${ }^{\mathrm{e}}$ siècle. Comme ses prédécesseurs Jacques de Vitry ou plus encore Étienne de Bourbon, Nider en accentue en effet la fonction probatoire ; la crédibilité est nécessaire à la théologie morale du Formicarius, explique-t-il dans le prologue, non certes à la manière de la vérité apodictique qui s'obtient par démonstration, mais selon la fonction inductive des faits probables ${ }^{21}$. Mais l'influence de cette tradition rationnelle n'est pas seule en cause. On peut penser que le contexte spirituel du premier tiers du $X v^{\mathrm{e}}$ siècle a joué dans le même sens : du temps de Nider, les lecteurs de littérature religieuse réclamaient de plus en plus de preuves visibles et publiquement vérifiables du surnaturel. Il y allait de l'efficacité du Formicarius. Dans l'esprit du dominicain, il ne s'agissait pas seulement d'offrir, avec ces quatre récits, des historiettes divertissantes, mais de proposer une intelligence critique des événements susceptible de redonner au présent de l'Église sa valeur d'histoire sainte.

La mise en forme agumentative des exempla était cependant un processus complexe, qui devait composer avec le statut hybride du genre et la présence d'éléments parasites. Il en est des deux premiers récits comme des histoires de saints analysés par A. Kleinberg : ils charrient des matériaux équivoques que la théologie n'assimile qu'imparfaitement ${ }^{22}$. Il est par exemple frappant de constater que, même s'ils servent le bien de l'Église et de la Chrétienté,

20. Dans C. Brémond, J. Le Goff, J.C. Schmitt, L' Exemplum », Turnhout, 1982 (Typologie des sources du Moyen Âge occidental, 40), p. 37-38. On trouvera une mise au point bibliographique dans J. Berloz et M.A. Polo De Beaulieu (dir.), Les exempla médiévaux. Nouvelles perspectives, Paris, 1998.

21. "Verum quia in morali nunc laboro materia, precor ideo lectorem sequentium ne demonstrationem querat ubi accomoda sufficit probabilitas »(prologue, page non numérotée).

22. A. Kleinberg, Histoires de saints. Leur rôle dans la formation de l'Occident, trad. française, Paris, 2005. 
ces exempla se déroulent hors de tout contexte religieux. Ils ne sont accompagnées d'aucune prière et ne font intervenir ni reliques ni saints; ils ne supposent même pas la foi chrétienne, le héros du premier exemplum étant juif. Indirectement, le statut théologique des événements rapportés s'en trouve affecté. Car ces anecdotes que Nider présente comme miraculeuses correspondent-elles tout à fait à ce qu'on entendait à l'époque par miracle ? À en juger par la définition classique due à saint Thomas d'Aquin comme par les règles retenues lors des procès de canonisation, on peut en douter ${ }^{23}$. Rien dans la dextérité de l'artilleur ne dépasse la puissance de la nature. La guérison de l'assiégé idiot ne revêt pas non plus le caractère d'immédiateté (il se remet en plusieurs jours et non instantanément) ni de perfection (il se nourrit en avalant une bouchée dans la cuisine) requis. Qui pis est, on ne peut s'empêcher de trouver le bilan de ces quatre miracles bien maigre : pas de victoire militaire, un seul mort du côté hussite et aucune conversion ! Au regard des critères médiévaux eux-mêmes, ces histoires relèvent en fait plus de la merveille que du miraculeux proprement dit. Nider semble avoir été conscient du problème. Tout en voyant dans ces événements la main de Dieu, il trahit un certain embarras pour les qualifier. Alors que son interlocuteur le Paresseux demandait des miracula, le Théologien préfère éviter le terme ; de manière plus vague, il parle de mirum, de mirabile, voire de spectaculum, faisant glisser le surnaturel du côté de l'esthétique ${ }^{24}$. C'est dire quelles tensions internes subsistent entre les critères du vrai, les structures de la narrativité orale et les exigences de l'apologétique.

Le travail de Jean Nider n'a donc pas échappé aux incertitudes et aux contradictions inhérentes à la littérature exemplaire. De longueur très inégale et empruntés à des sources diverses, ces quatre exempla sont le fruit de transactions plus ou moins habiles entre des formes d'écriture et de raisonnement qui ne s'harmonisent pas. De telles discordances n'en sont pas moins précieuses pour l'historien, car elles laissent échapper des renseignements irremplaçables sur les réalités du combat contre les Hussites.

\section{Des histoires vraies ?}

Ce serait faire injure à Nider que de ne pas le prendre au mot lorsqu'il situe les anecdotes qu'il rapporte et met en scène, non des quidams de toute espèce évoluant sub specie aeternitatis, mais des personnes en chair et en

23. Lex explications de saint Thomas se trouvent dans sa Summa Theologiae, I q. 105 a. 7. Sur les critères du miraculeux en matière de canonisation, voir A. VAuCHEZ, La sainteté en Occident aux derniers siècles du Moyen Âge, Rome, 1981, p. 40 et sv.

24. «Ibi mirum tale... evenerat » (p. 254) ; « altera die mirabile aliud contigit... ( (p. 255); « spectaculum tunc Deus mirantibus omnibus perfidis... » (p. 257). Sur la distinction et les flottements entre miraculeux et merveilleux, voir Miracles, prodiges et merveilles au Moyen Âge (25 congrès de la SHMESP), Paris, 1995. 
os. Les informations transmises sont-elles véridiques ? Penchons-nous sur les circonstances de ces quatre exempla de manière à en évaluer la fiabilité : encore que par prudence le dominicain taise souvent le nom des protagonistes ${ }^{25}$, son témoignage offre une riche matière qui se laisse recouper avec d'autres sources.

Que s'est-il réellement passé lorsque les guerriers hussites se furent emparés de la petite ville silésienne de Frankenstein (Zạbkowice Sląskie) ? L'événement date de la contre-offensive de 1428, qui porta des troupes d'Orphelins dans la région de Glatz (Kladsko) ${ }^{26}$. Début avril, ceux-ci trouvèrent à Frankenstein un couvent de Frères Prêcheurs que tout désignait à leur vindicte : non seulement c'étaient des religieux, coupables à ce titre de préférer leurs traditions humaines à la la pureté de l'Évangile, mais ils appartenaient à l'ordre qui depuis des années se faisait gloire de pourfendre avec la dernière énergie les erreurs wycliffistes et hussites ${ }^{27}$. De surcroît, les Frères étaient, à l'instar de leurs concitoyens de Frankenstein, germanophones... Comme tant d'autres religieux de Bohême et des pays voisins, les dominicains de Frankenstein furent donc acculés à ce choix dramatique : l'apostasie ou la mort. Écrivant vers 1430, Hermann Korner, suivi par l'auteur anonyme de la Chronique dite de Rufus, confirme que le sous-prieur du couvent fut alors livré aux flammes ${ }^{28}$. Nider, qui ne dépend pas de ces deux sources lubeckoises, ignore ce qui y est dit de l'emprisonnement du prieur ainsi que de l'acte iconoclaste prêté aux Hussites. Mais il n'en fallait pas plus pour que la postérité ne se saisît de ces événements sanglants. Les reliques de notre martyr furent pieusement conservées à Frankenstein; une tradition locale, malheureusement difficile à dater, lui donna le nom de Nicolas Carpentarii et lui adjoignit deux autres compagnons d'infortune, Jean Buda et

25. Nider se conforme là à un usage ancien, qu'il justifie en ces termes dans le prologue : «Nec miretur quis si quandoque loca et nomina propria personarum siluero, quia ita fieri debere fraterna caritas imperat, et recta persuadet ratio, ubi aut confessionis sigillum, aut secretum aliquod notabile, aut periculum cenodoxie ad aliqua silenda adstrinxit, vel periculum aliud corporis aut anime » (page non numérotée).

26. Le détail des événements est donné par C. Grünhagen, Hussitenkämpfe der Schlesier 1420-1435, Wrocław, 1872, p. 146. Il faut corriger C. Chène qui date l'événement de 1430 (article cité, p. 335).

27. Sur le couvent de Frankenstein, cf. J. Kloczowski, «Dominicans of the Polish Province », dans ID. (dir.), The Christian Community of Medieval Poland, Wroclaw, 1981, p. 73118 (ici carte p. 101). C'est par erreur que C. Chène situe l'événement dans « un couvent réformé de Bohême » (article cité, p. 337). Sur l'opposition entre Hussites et Frères mendiants, cf. O. MARIN, L'archevêque, le maître et le dévot. Genèses du mouvement réformateur pragois (années 1360-1419), Paris, 2005, deuxième partie.

28. «Se quemen sundergen in das closter to Frankensteen der predeker orden unde begunden das to vorsterende ; des straffede se de prior zwarliken umme ere undaet ; den guden innighen broder nemen se unde brochten ene in de kerken; dare sammelden se to hope de bilde der hilgen unde makeden dar en wu raf unde worpen den supprior dar in unde vorbranden ene » (éd. citée, p. 308). La version D de la Chronica novella d'Hermann Kolner indique seulement : «Suppriorem quoque fratrum Predicatorum de conventu Frankenstein, virum valde devotum, igne facto de sanctorum ymaginibus incineravit » (éd. citée, p. 492). 
André Cantoris. Puis la Contre-Réforme en célébra solennellement le souvenir, de sorte que jusqu'à nos jours, des démarches ont été tentées pour promouvoir leur béatification ${ }^{29}$. Force est cependant de constater la fragilité du dossier. S'il n'est pas question de mettre en doute l'exécution du sous-prieur, on peut en revanche soupçonner que Nider et ses confrères ont enjolivé sa mort pour les besoins de leur cause. L'historien ne peut guère s'aventurer sur ce terrain, faute de sources extérieures à l'ordre dominicain.

Nous sommes heureusement mieux renseignés sur les circonstances dans lesquelles la délégation conciliaire échappa à l'épidémie de peste dont Prague était la proie. Cette deuxième ambassade séjourna sur les rives de la Vltava du 22 octobre 1433 au 14 janvier 1434. Nider l'a allègrement confondue avec la première ambassade, celle qui avait été dépêchée par le concile à l'été précédent. Contrairement à ce qu'il écrit, celle-là ne comprenait pas l'évêque d'Augsbourg Pierre de Schaumberg ni de «nombreux autres docteurs » : seuls furent du voyage Philibert, évêque de Coutances, Jean Palomar, archidiacre de Barcelone, le doyen de Tours Martin Berruyer et le chanoine de Magdebourg Henri Toke ${ }^{30}$. Pour le reste, sa version des faits rejoint celle qui fut présentée au concile. Les membres de la délégation revinrent en effet tous sains et saufs à Bâle et purent décrire comment ils avaient été épargnés par les assauts de la peste qui décimait les Pragois autour d'eux. À leur suite, Nider commet au moins un mensonge par omission : il passe sous silence l'aide que leur prodiguèrent les docteurs en médecine de l'université pragoise sous forme de médicaments et de prescriptions ${ }^{31}$. De plus, la fin heureuse de cette ambassade est moins extraordinaire que ne le pensaient l'auteur du Formicarius et ses contemporains. Les démographes s'accordent ajourd'hui à penser que la peste de 1433 n'en était pas une. Sous ce terme générique se dissimule une épidémie de typhus provoquée par les mauvaises récoltes des années précédentes ${ }^{32}$. Or on sait que la propagation de ce virus dépend largement des conditions socio-économiques, notamment de la sous-alimentation qui en multiplie la létalité par deux ou par trois. Il n'est donc pas nécessaire d'ajouter foi à l'idée d'une protection divine particulière : les représentants conciliaires durent bien davantage leur salut au confort dont ils bénéficièrent. Ils furent moins chanceux quand la

29. Typique de cette orientation est le travail d'A. Neumann, Die katholischen Märtyrer der Hussitenzeit, Warnsdorf, 1930 (ici p. 54-61). L'ouvrage du dominicain H. WILMS, Die Märtyrer von Frankenstein, Oldenburg, 1934, ne m'a pas été accessible.

30. Voir V.V. Tomeк, Dějepis města Prahy, Prague, 1879, t. 4, p. 607-622.

31. Voir le témoignage du Liber de legationibus, éd. J. BIRK, Monumenta conciliorum generalium saeculi decimi quinti, Vienne, 1857, t. 1, p. 448 : «Sed et magistri medicine, multum eis timentes, dederunt contra pestem, que Prage vigebat, remedia et in scriptis et de apoteca». L'auteur de cette œuvre est probablement Jean de Tours, secrétaire de l'évêque Philibert, comme l'a suggéré F.-M. BARToš, Husitská revoluce, t. 2, Prague, 1966, p. 158. L'université de Prague était réputée pour ses consilia contra la peste : cf. K. SudHoff, «Prager Pesttraktate aus dem 14. Jahrhundert », Archiv für Geschichte der Medizin, 7, 1913, p. 57-114.

32. J. Vlk (dir.), Dějiny Prahy, t. 1, Prague, 1997, p. 255. 
peste se déclara - cette fois-ci pour de bon - en 1439 à Prague : le 19 juin, elle emporta le plus éminent d'entre eux, l'évêque Philibert...

Les deux premiers exempla posent enfin un problème spécifique, dans la mesure où il n'existe pas de source contemporaine qui documente le siège de Plassemburg. Tous les autres témoignages datent de l'époque moderne et grossissent l'ampleur des destructions causées. Quel crédit accorder à ce qu'en écrit Nider? L'événement prend place lors des « magnifiques chevauchées » (spanilé jizdy) qui, en 1429-1430, virent les guerriers hussites ravager les régions allemandes de l'Empire. Tout ce que l'on sait de précis dans ce cas est qu'après la prise de Hof et Münchberg à la mi-janvier 1430, les Orebites se séparèrent du gros de la troupe ; emmenés par Jíra de Řečice, ils investirent le $31 \mathrm{Kulmbach}$, mais durent lever le camp après une attaque mal engagée contre Plassemburg et rejoignirent les Taborites qui marchaient sur Bamberg ${ }^{33}$. À ce scénario, le Formicarius ajoute plusieurs éléments dont il faut apprécier la vraisemblance.

Il y a d'abord la présence d'assiégés juifs, l'un artilleur, l'autre chirurgien. Nider ne l'a pas inventée. Une communauté juive est bel et bien attestée dans la ville entre 1373 et 1515 , date de son expulsion. Jusque-là, elle avait été placée sous la protection du margrave de Brandebourg, disposait d'une école et donna son nom à une rue ${ }^{34}$. De plus, le tableau que brosse le dominicain des relations entre voisins juifs et chrétiens n'a rien que de très vraisemblable. Comme le suggère le deuxième exemplum, il devait être courant, à Kulmbach comme ailleurs, de faire appel aux services des médecins et chirurgiens juifs : beaucoup de chrétiens, à commencer par les clercs, préféraient bénéficier de leurs talents plutôt que de se plier aux interdictions martelées en vain de synode en synode. Nider considère donc à bon droit comme exceptionnel le refus qu'oppose le blessé au chirurgien juif venu le soigner ${ }^{35}$. Quant à l'intervention de l'artilleur juif dans le premier exemplum, elle témoigne que l'identité civique transcendait à Kulmbach les clivages confessionnels. Sous la plume d'un clerc qui ne manifeste par ailleurs aucune sympathie pour le judaïsme, l'anecdote mérite d'être prise au sérieux. De fait, la présence d'un Juif au combat n'avait rien d'impossible si l'on veut bien se rappeler que malgré les restrictions progressives, beaucoup d'entre eux continuaient dans l'Allemagne de la fin du Moyen Âge à porter les armes. Aussi pouvaient-ils participer quand l'occasion s'en présentait à la défense de leurs cités ${ }^{36}$. Le récit qu'en fait Nider s'avère d'autant plus inté-

33. D'après G. SChlesinger, Die Hussiten in Franken. Der Hussiteneinfall unter Prokop dem Grossen im Winter 1429/30, seine Auswirkungen sowie sein Niederschlag in der Geschichtschreibung, Kulmbach, 1974.

34. Voir la notice consacrée à Kulmbach dans Germania judaica, 3/1, Tübingen, 1987, p. 684-696.

35. Sur cette question, cf. J. Shatzmiller, Jews, Medicine and Medieval Society, BerkeleyLondres, 1994.

36. Comme l'a montré G. Kisch, The Jews in Medieval Germany, Chicago, 1949, p. 119 et sv. 
ressant qu'il prend le contre-pied des rumeurs de complot judéo-hussite qui avaient longtemps circulé en Bohême comme en Autriche : à Vienne en 1419-1420, puis à Jihlava (Iglau) en 1426, on avait accusé sans preuve les Juifs de porter assistance aux hérétiques ${ }^{37}$. Sur cette histoire complexe, encore mal connue des historiens, notre exemplum projette un éclairage bien différent de celui des amalgames et des préjugés ambiants.

La destruction de la statue du Christ telle que la rapporte l'auteur du Formicarius nous fournit un autre moyen de mettre à l'épreuve sa fiabilité. Nider donne en effet une indication précieuse lorsqu'il nous apprend que la statue représentait Jésus au Mont des Oliviers, en prière et les bras levés. Les historiens de l'art ont depuis longtemps montré quel engouement ce motif tiré des évangiles synoptiques, mais rare jusque là dans l'iconographie chrétienne, avait connu au déclin du Moyen Âge. La chronologie et la géographie de sa diffusion concordent avec ce qui en est dit ici : les plus anciennes statues conservées datent des années 1420 et se trouvent en Franconie, dans les régions de Wurzbourg et de Nuremberg. L'église de Veitlahn, à quelques kilomètres de Kulmbach, en abrite encore ajourd'hui un bel exemplaire, en pierre, du milieu du siècle ; comme souvent, le Christ y est intégré dans un ensemble monumental, qui comprend l'ange et le groupe des apôtres endormis ${ }^{38}$. La fonction de ces groupes sculptés était de proposer aux fidèles le spectacle d'un ars moriendi évangélique et de les faire compatir à l'agonie du Christ sauveur. Aussi étaient-ils souvent placés sur le côté sud des églises, près du cimetière, dans une relation de protection à l'égard des défunts de la communauté.

C'est donc très vraisemblablement une statue de ce type que les guerriers orebites ont sortie de l'église paroissiale Saint-Pierre de Kulmbach avant de la décapiter. De tels actes iconoclastes n'étaient pas à l'époque réservés aux seuls Hussites. Comme l'a montré G. Marchal, il n'était pas rare que les armées en campagne s'en prennent ainsi aux symboles les plus chers de l'ennemi, et notre soldat n'y fait pas exception : en détruisant cette statue, il tourne en dérision le capital sacré de Kulmbach ${ }^{39}$. Mais le hussitisme avait donné un sens nouveau à ces anciens gestes de provocation. Ainsi que le relève Jean Nider au chapitre 9 du troisième livre, les Bohémiens détruisaient systématiquement les statues des saints, convaincus qu'ils étaient de leur illicéité théologique : l'enjeu tournait désormais autour de de la présence du

37. Sur ces soupçons, voir F. ŠMAHEL, Die hussitische Revolution, op. cit., p. 1722-1723. Certains Juifs de l'Empire y ajoutèrent eux-mêmes foi et imaginèrent que les Hussites avaient renié la Trinité : cf. I. J. YuvaL, « Juden, Hussiten und Deutsche. Nach einer hebräischen Chronik », Zeitschrift für historische Forschung, Beiheft 13, Berlin, 1992, p. 59-102.

38. On dispose sur le sujet de la thèse de D. MunK, Die Ölberg-Darstellung in der Monumentalplastik Süddeutschlands, Erlangen, 1968, ici $\mathrm{n}^{\circ} 385$.

39. Cf. G. MARChAL, «Jalons pour une histoire de l'iconoclasme au Moyen Âge », Annales ESC, 50, 1995, p. 1135-1156. 
saint dans ses représentations figurées ${ }^{40}$. Le caractère récent de l'iconographie du Mont des Oliviers et son association avec la prière pour les morts auraient-ils particulièrement excité l'ire des guerriers hussites ? Peut-être. En tout état de cause, la profanation de la statue de Kulmbach peut être rapprochée de nombreux autres actes iconoclastes, tels que ceux qui eurent lieu au château de Prague en juin 1421 et dont Laurent de Březova a conservé le souvenir. Les partisans de Jean Želivský avaient alors littéralement exécuté la statue du Christ des Rameaux, selon un rituel de dérision en tout point semblable au nôtre : même apostrophe railleuse, même mise à mort symbolique $^{41}$.

On le voit, la convergence de ces éléments laisse penser que nous n'avons pas affaire ici à une simple projection idéologique, mais à une version des faits somme toute bien informée, quoique naturellement écrite dans un esprit militant. Les préventions de Nider à l'encontre du hussitisme ont bien pu lui en dissimuler l'inspiration réformatrice authentique, elles ne l'ont certes pas empêché de faire un travail de terrain rigoureux. Rien d'étonnant dès lors à ce que, dans cette bourse galopante à l'information qu'était le concile de Bâle, le dominicain ait pu être invité à lire publiquement les bonnes feuilles de l'œuvre qu'il était en train de composer ${ }^{42}$.

\section{Les fonctions du miraculeux}

En plus de nouvelles fraîches, le public du concile se montrait avide de recevoir des enseignements édifiants. C'est précisément à l'aune de cette visée qu'il reste à décrypter les significations symboliques de nos quatre exempla. À cet égard, Nider ne tire pas explicitement la leçon des histoires qu'il raconte et laisse au lecteur le soin de le faire. Il est vrai que toutes ces

40. «Nihil de imaginibus sanctorum, nec de ornamentis ecclesiarum tenebant, sed omnia ista destruxerunt, et illicita esse esse dixerunt» (p. 239). Sur l'iconoclasme hussite dans sa dimension pratique comme théorique, voir en dernier lieu N. SCHNITZLER, Ikonoklasmus Bildersturm. Theologischer Bilderstreit und ikonoklastisches Handeln während des 15. und 16. Jahrhunderts, Munich, 1996.

41. "Tercia autem feria post ymagines magistrales et tabule altarium plurimum subtiles et preciose ad Johannis presbiteri induccionem cum blasphemia sunt combuste et inter cetera, ut eorum plus patens foret vesania, ymaginem Christi asello insidentem in cimboriis exponunt ecclesie et versa ad Misnam facie blasphemando dicunt : 'Tu, si es Christus, benedicas Misnam'. Et statim trudentes de cimboriis ipsam in pecias confringunt » (Chronica hussitarum, éd. J. Goll, dans Fontes Rerum Bohemicarum, t. 5, Prague, 1893, p. 484).

42. Les témoignages en ce sens ne remontent toutefois pas en deçà du XVI $I^{\mathrm{e}}$ siècle : voir W. Tschacher, op. cit., p. 84-85. Sur les formes de la communication au concile de Bâle, on se reportera à l'étude devenue classique de J. MieThKe, « Die Konzilien als Forum der öffentlichen Meinung im 15. Jahrhundert », Deutsches Archiv, 37, 1981, p. 736-773, qu'on complètera à l'aide de J. HelmRath, «Kommunikation auf den spätmittelalterlichen Konzilien », dans H. PoHL (dir.), Die Bedeutung der Kommunikation für Wirtschaft und Gesellschaft, Stuttgart, 1989, p. 116-172. 
anecdotes délivrent à première vue un message simple, d'ailleurs commun au genre de l'exemplum dans son ensemble : la punition des méchants (le profanateur hussite est tué) et la récompense des justes, par l'octroi de la guérison (le simple d'esprit), de la protection (les représentants du concile) ou encore de la palme du martyre (le sous-prieur de Frankenstein). La « lutte du Bien contre le Mal» (C. Chène) livre-t-elle pourtant le fin mot de ces histoires? Ne peut-on pas aller plus loin et tenter de percer quelles fonctions, diverses et souvent équivoques, remplit à chaque fois le miraculeux ?

La plus évidente est la fonction de légitimation. Le troisième exemplum l'illustre parfaitement : au travers de son court récit, Nider cherche à exalter l'intégrité, tant morale que doctrinale, du concile de Bâle. Il le fait d'abord en soulignant l'humilité du comportement adopté par ses représentants à Prague. Le dominicain se fait ici l'écho de préoccupations largement partagées par les Pères, qui se souvenaient du piteux effet que le faste et les désordres de certains clercs avaient produit sur les partisans de Jean Hus à Constance; des consignes furent passées pour éviter que ne se réitère ce malheureux précédent ${ }^{43}$. Contre le chantage à la fidélité évangélique des Hussites, cet exemplum fait donc valoir que les délégués catholiques n'étaient pas moins simples et dépouillés que leurs adversaires bohémiens. Mais c'est plus encore l'orthodoxie du concile que soutient le Formicarius. Des trois fléaux bibliques qui, d'après les témoins, s'étaient abattus sur la Bohême, la faim, la peste et le glaive, Nider ne retient que le deuxième - et pour cause : depuis l'époque patristique, la maladie était devenue la métaphore obligée de l'hérésie. R. I. Moore a ainsi montré comment aux XII et XIII siècles la référence à la lèpre émailla la littérature anti-hérétique et servit à ses auteurs de modèle systématique pour à la fois penser la propagation de l'hérésie et proposer des mesures de guérison ${ }^{44}$. Passé le milieu du XIV ${ }^{\mathrm{e}}$ siècle, la peste reprit toutefois la première place dans l'imaginaire de l'Occident. Aux yeux de Nider, la rapidité de sa diffusion et sa malignité n'étaient assurément pas inappropriées pour traduire le succès du hussitisme : l'infection des corps pestiférés renvoyait immédiatement à la souillure des âmes égarées. Cette anecdote ne fait donc pas que raconter un banal miracle de protection. Sur un mode allégorique aisément accessible, elle met en scène l'incorruptibilité de la foi de l'Église et de ses porte-parole autorisés, qu'elle oppose aux errements dévastateurs des hérétiques.

43. Le 4 juin 1432, les Pères avaient ordonné à quiconque appartenait au concile de se réformer dans son costume comme dans le reste, afin de ne laisser aux Tchèques aucun sujet de scandale (Monumenta conciliorum generalium..., t. 1, éd. citée, p. 224). Le modus vivendi in concilio s'inscrit dans ce même contexte. Relevons pour notre propos qu'il stipule : «Et quia qualis paterfamilias tales et domestici eius, curandum est, ut non solum patres de concilio, sed eorum familiares decentem honestatem servent... » (éd. J. MANSI, Sacrorum conciliorum nova et amplissima collectio, t. 30, repr. Graz, 1961, col. 253).

44. Dans son article «Heresy as Disease », dans W. Lourdaux et D. Verhelst (dir.), The Concept of Heresy in the Middle Ages (11th-13th C.), Louvain-La Haye, 1976, p. 1-11. 
Le quatrième exemplum remplit, quoique de manière plus subtile, une fonction analogue. La figure de style employée ici est celle de la prétérition. Tout en se défendant d'en parler, Nider attire l'attention sur les mérites de ce Frère Prêcheur et sur la gloire qui en découle pour l'ordre tout entier. Le sous-prieur se voit aussitôt érigé en parangon des vertus de patience, de dévotion et de fidélité à la règle, celles-là même que Nider cherchait à rétablir dans les couvents ralliés à l'observance. Convaincu qu'une des causes de l'hérésie hussite venait du fait que religieux et religieuses ne s'étaient pas réformés, l'auteur du Formicarius trouvait là le moyen d'enfourcher son grand cheval de bataille ${ }^{45}$. C'était en même temps pour lui l'occasion de réveiller l'ardeur du martyre qui avait été un des charismes primitifs de son ordre. Saint Dominique n'avait-il pas rêvé de s'exposer aux dangers de l'évangélisation des Cumans païens? Certains de ses premiers disciples n'avaient-ils pas péri sous les coups des cathares? Or l'histoire allait se répétant. Les Frères Prêcheurs venaient de payer un lourd tribut à la révolution hussite. À l'instar de Nider, chroniqueurs et prédicateurs dominicains se plurent à mettre en avant la cohorte de ces nouveaux martyrs. En ce premier tiers $\mathrm{du} \mathrm{XV}^{\mathrm{e}}$ siècle, l'Europe centrale avait depuis longtemps supplanté le Midi albigeois comme terre d'élection de l'héroïsme dominicain, et cet exemplum participe à un tel basculement ${ }^{46}$.

Par là, Nider réagissait aussi à la crise que traversait à la fin du Moyen Âge la notion de martyre. Que l'auteur du Formicarius assimile la mort du sous-prieur à un quasi miracle en dit long sur la raréfaction de cette forme de sanctification: depuis 1253, l'Église n'avait plus canonisé le moindre martyr, de sorte que le prestige attaché à la mort violente était en passe de devenir le seul fait des dissidents et des hérétiques ${ }^{47}$. Les Hussites, en particulier, en revendiquaient le monopole et vénéraient bruyamment leurs martyrs les plus célèbres, Jean Hus et Jérôme de Prague. À Constance, le courage des deux Tchèques avait d'ailleurs ému plus d'un spectateur catho-

45. Nider écrivit ainsi aux religieuses de Schönensteinbach en 1428 : «Nun sprechen dy laien... münch vnd nonne thünt kein güt. Die hussen verderben sie dann cze grund » (cité par M. BRAND, Studien zu Johannes Niders deutschen Schrifen, Rome, 1998, p. 234).

46. Comme l'a bien montré A. Reltgen-TALlon, «Vers un autre Sud? Les marges orientales de la chrétienté comme nouvelle terre de mission dans l'imaginaire dominicain du Moyen Âge », dans Tous azimuts. Mélanges G. Jehel, Histoire médiévale et archéologie 13, 2002, p. 411-422. Voir en particulier la chronique d'Albert de Castello, qui rapporte ici le témoignage de Léonard d'Udine (dans E. MARTENE et U. DuRAND, Veterum scriptorum... collectio, tome 6 , 1729, col. 381) : «Propter hanc causam plures fratres nostri martyrio coronati sunt ab hereticis ; et dum essem in concilio Ferrariensi tempore domini Eugenii pape IV, relatum est plures conventus nostri ordinis funditus eversos ab Hussitis in Bohemia, et plures trucidatos fratros ab eisdem. Ordo enim noster valde insudavit contra hereses ad earum exstirpationem, et precipue circa hoc sacramentum Corporis Christi. Hec magister Leonardus predictus ».

47. On trouvera une vue d'ensemble sur le phénomène dans M. Rubin, « Choosing Death. Experiences of Martyrdom in Late Medieval Europe », dans D. Wood (dir.), Martyrs and Martyrologies, Oxford, 1993, p. 185-208. Sur les restrictions de la politique de canonisation dans ce domaine, on lira A. VAuCHEZ, La sainteté en Occident..., op. cit., p. 482 et sv. 
lique ; quelques années plus tard, les soldats hussites impressionnèrent à leur tour leurs adversaires par leur mépris de la mort ${ }^{48}$. Dans ces conditions, il est probable qu'avec ce récit, Nider ait cherché à concurrencer les hérétiques sur leur propre terrain. L'exemple du sous-prieur de Frankenstein répond à une urgence, celle de prouver que l'Église se montre toujours capable de donner d'authentiques martyrs comme elle l'avait fait aux premiers temps du christianisme. On peut toutefois se demander si pareille apologie rencontra quelque écho. Même au sein de l'ordre dominicain, il semble bien que le sous-prieur de Frankenstein n'ait bénéficié d'aucune reconnaissance officielle. On le chercherait par exemple en vain dans les catalogues de martyrs comme dans les vies des frères illustres qui fleurirent à l'époque ${ }^{49}$. La méfiance qu'éprouvaient les hautes sphères de l'Église à l'endroit du martyre limitèrent donc l'efficacité qu'attendait Nider de cet exemplum édifiant.

Il est plus difficile de dégager des deux autres exempla une leçon claire et sans équivoque. Quels qu'en soient les fondements réels, le propos a certainement, comme l'a reconnu C. Chène, une finalité polémique. Faire par exemple du Juif le vengeur du Christ outragé revient à présenter les Hussites comme des déicides pires que les scribes et les pharisiens eux-mêmes. Dans cette répétition symbolique de la Passion, ce sont eux qui jouent le rôle des bourreaux et qui attirent de ce fait la malédiction. L'auteur du Formicarius réactualise ainsi à mots couverts un soupçon maintes fois porté contre les hérétiques. Depuis des siècles, nombreux étaient les polémistes qui reprochaient aux dissidents de tout poil de judaïser ${ }^{50}$, et les Hussites n'échappèrent pas à cette accusation; leur iconoclasme, en particulier, y donnait matière. L'exemplum s'inspire donc d'un vieil argument de controverse, à ceci près que Nider en tire un éloge pour le moins inattendu de ce Juif impavide. À cet égard, le récit tranche vigoureusement sur les stéréotypes anti-juifs qui imprégnent la littérature exemplaire de l'époque. D'ordinaire en effet, les Juifs n'y font que de la figuration, leur rôle consistant à causer involontaire-

48. Parmi d'autres exemples, voir sur le versant français le sermon que Jean Gerson prononça le 7 juin 1416, peu après la mort de Jérôme (sermon Spiritus Domini, dans L'Euvre oratoire, éd. P. GlorieuX, Paris, $1963, \mathrm{n}^{\circ} 247$, p. 537) : «Denique si quis posset scire certitudinaliter de se vel altero quod habeat Spiritum Sanctum, cum videamus haereticos promptos ad moriendum plus aliquando quam fideles catholicos, et unde procedit? ». La Chronique d'Enguerrand de Monstrelet va dans le même sens (éd. L. Doü̈T d'ARCQ, Paris, 1860, t. 4, chapitre 259, p. 86) : «Et pour vray, iceulx mauldis hérétiques estoeint si obstinez en leurs erreurs qu'ils ne craignoient nulz martires dont on les feist mourir ». Le passage est démarqué de la Chronique dite des Cordeliers (ibid., t. 6, Paris, 1862, p. 310) : «Et furent ces hérites sy fort obstinez en leur erreur que ils ne crémoyent nul martire ne nulle mauvaise mort dont on les peuist faire morir ».

49. Ont été dépouillés les documents édités par R. LOENERTZ, «Un catalogue d'écrivains et deux catalogues de martyrs dominicains », Archivum Fratrum Praedicatorum, 12, 1942, p. 279-303, ainsi que le Liber de viris illustribus dû à Jean Meyer (éd. P. von Loë, Leipzig, 1918).

50. Sur les lieux parallèles entre la littérature anti-hérétique et la controverse antijuive, voir G. Dahan, Les intellectuels chrétiens et les juifs au Moyen Âge, Paris, 1990, p. 362-366. 
ment le salut des chrétiens, quand ils ne sont pas eux-mêmes gibier de baptême ${ }^{51}$. Or voici que dans l'exemplum le Juif apparaît au contraire comme le héros de l'action : il est celui qui venge l'honneur de Kulmbach, qui agit au nom de la communauté entière tout en demeurant fidèle à sa foi. Comment donc comprendre que cet habituel bouc-émissaire des communautés chrétiennes soit ici érigé en sauveur?

La comparaison avec l'exemplum suivant suggère de voir là un mécanisme de compensation, destiné à contourner les problèmes apparemment insolubles qui se posaient à une Église désemparée face aux succès hussites. Avec ce deuxième récit, nous sommes en présence, non d'un fou proprement dit, mais d'un simple d'esprit que son arriération de naissance a mis à part de la communauté, tout comme la religion distingue l'artilleur de ses voisins chrétiens. Or son courage et son ardeur au combat ont beau être insensés, cela lui vaut d'être qualifié par Nider de «petit pauvre du Christ». L'expression n'est pas choisie au hasard: elle indique que cette victime innocente des Hussites s'est unie par ses souffrances et ses blessures à la Passion du Seigneur. L'histoire illustre de la sorte l'inversion des hiérarchies et des statuts dont Nider fait un leitmotiv de son œuvre. Dès le prologue, pour se justifier de citer souvent en exemple de simples femmes, le dominicain paraphrase le mot de l'Apôtre selon lequel Dieu choisit ce qui est faible dans le monde pour confondre les forts ${ }^{52}$. Dans une Église qui peine à restaurer son autorité, la faiblesse et la marginalité apparaissent ainsi comme les instruments privilégiés du miraculeux. Telle est la fonction paradoxale de ces deux récits. Alors que les autres exempla confortent la légitimité des autorités religieuses en place, qu'il s'agisse des Pères conciliaires ou des Frères Prêcheurs, ceux-ci ne rétablissent l'ordre de la justice divine qu'au moyen d'une suspension des médiations et des hiérarchies ordinaires : ils donnent le premier rôle aux exclus. Gardons-nous certes de prêter au dominicain une intention subversive. S'il fait du Juif et de l'idiot les derniers recours contre les armées hussites, le but de Nider reste bien, en dernier ressort, de faire honte à un peuple chrétien indifférent et couard, dont les chevaliers ont tourné bride à la première escarmouche. Il n'en est pas moins significatif de voir la lutte contre l'hérésie justifier, ne serait-ce que momentanément, des entorses aussi inouïes à la norme que les pouvoirs auraient voulue irrécusable. Joue ici à plein le caractère intrusif du miraculeux, dont l'imprévisibilité demeure rebelle à tout ordonnancement social ou religieux.

51. J. LE Goff en a retracé les principales caractéristiques dans son article «Le Juif dans les exempla: le cas de l'Alphabetum narrationum », dans M. OLENDER (dir.), Pour Léon Poliakov. Le racisme, mythes et sciences, Bruxelles, 1981, p. 209-217.

52. « Nec me suspicetur faciliter credere hominibus quibuslibet, presertim feminis, quas nisi sint probate, plurimumsemper in talibus delirare suspicor, quanquam sexus non despiciendus sit fragilis, in quo sexu Deus sepe magna fecit ut confundat fortia mundi secundum Apostolum (1 Co 1, 27)» (page non numérotée). 
Le Paresseux a-t-il été satisfait des exempla allégués par le Théologien ? La fin abrupte de la troisième partie du Formicarius ne permet pas de le savoir. Toujours est-il que ce chapitre livre l'une des premières tentatives rétrospectives pour fonder sur des manifestations miraculeuses une apologétique anti-hussite. Tentative prématurée et encore bien embarrassée : Nider en est réduit à citer en exemple des personnages obscurs dont les «miracles » apparaissent plus que problématiques, quand ils ne contreviennent pas à l'orthopraxie. La spiritualisation de l'histoire à laquelle procède le dominicain bute là sur une évidente carence. Autant l'Église réunie en concile a su trouver les moyens intellectuels et diplomatiques de répondre à la contestation hussite, autant les charismes de la sainteté lui ont fait défaut ; elle n'a pas vu se lever en son sein de nouveaux saint François et saint Dominique, capables d'opposer aux séductions de l'hérésie le témoignage de leur vie ascétique et de leurs pouvoirs surnaturels. Quinze années plus tard, ce sera le rôle de saint Jean de Capistran que de relever le défi, avec le sens de la théâtralité et la violence rhétorique dont il avait le secret ${ }^{53}$.

Resterait, pour prolonger cette interprétation, à examiner la postérité de nos quatre exempla anti-hussites. Il est certes garanti qu'ils ont largement circulé, insérés qu'ils étaient dans une œuvre à succès : les quelque vingtcinq manuscrits conservés et les trois éditions incunables du Formicarius les ont fait connaître des milieux cultivés, spécialement conventuels, de l'Allemagne du sud. Mais quelle en a été au juste la fortune ? Les prédicateurs auxquels Nider destinait prioritairement son œuvre les ont-ils réemployés et les ont-ils traduits en langue vulgaire ? L'étude de la réception du Formicarius n'en est hélas qu'à ses balbutiements. Elle éclairerait à coup sûr d'un jour nouveau cette histoire des réactions spirituelles au hussitisme qui reste largement à écrire.

Olivier Marin, CRESC, Université Paris-Nord, 99 avenue Jean-Baptiste Clément, F-93430 Villetaneuse

\section{Miracle et apologétique : sur quelques exempla anti-hussites dans le Formicarius de Jean Nider}

Le dominicain Jean Nider (1380-1438) participa activement au règlement de la question hussite à Bâle. Mais la réponse qu'il y apporta ne fut pas seulement diplomatique. Son traité de discernement des esprits, le Formicarius, vise entre autres à réduire le scandale que constituait aux yeux de beaucoup le succès, au moins partiel, de l'hérésie bohémienne. C'est ainsi que dans le dernier chapitre du troisième livre, Nider raconte quatre exempla censés prou-

53. Voir sur ce point les études de F. ŠMAHEL, «'Spectaculum fidei’ českomoravské mise Jana Kapistrana », Z kralické tvrze, 14, 1987, p. 15-19 et de K. Elm, « Johannes Kapistrans Predigtreise diesseits der Alpen (1451-1456) », dans idem, Vitas fratrum. Festgabe zum 65. Geburtstag (Saxonia Franciscana 5, 1994), p. 321-337. 
ver que Dieu n'a pas abandonné le peuple catholique en lutte contre les Hussites. L'étude cherche d'abord à en identifier les circonstances : les deux premiers récits se déroulent lors du siège de Kulmbach par les Orebites fin janvier 1430, le troisième a lieu à l'occasion de l'ambassade du concile à Prague à l'automne 1433, et le quatrième pendant le pillage du couvent dominicain de Frankenstein (Silésie) en 1428. On montre ensuite comment s'y déploient les ressources apologétiques du miraculeux et quelles en sont les incertitudes; pris entre le souci de légitimer les autorités religieuses et la nécessité de faire une place à l'intervention sauvage du surnaturel, Nider se débat dans des contradictions non résolues. Le Formicarius illustre ainsi indirectement les difficultés auxquelles étaient confrontés les théologiens du $\mathrm{XV}^{\mathrm{e}}$ siècle pour redonner au présent de l'Église sa valeur d'histoire sainte.

Hussitisme - Miracles - Jean Nider - Exempla - Bohême

\section{Miracle and apologetics : four exempla in Johannes Nider's Formicarius}

The Dominican Johannes Nider (1380-1438) played an active role in the settlement of the Hussite case in Basel. But the answer he gave was not only diplomatic. His treatise on the discernment of spirits, the Formicarius, aims among other things to subdue the scandal which had arisen from the partial success of Hussitism. For this reason, in the last chapter of the third book, Nider relates four stories (exempla) which are supposed to prove that God has not abandoned Catholics in their fight against the Hussites. The present paper tries first to provide the elements of the historical context : the first two stories occurr during the siege of Kulmbach by the Orebites at the end of January 1430, the third takes place when the conciliar legation stayed in Prague in the autumn of 1433 and the fourth during the plundering of the Dominican convent of Frankenstein in Silesia in 1428. These stories show how the miracle serves apologetic purposes and the uncertainties involved. Nider struggles with the contradictions, torn between his aim to legitimize religious authorities and the need to give a chance to the supernatural. In this respect, the Formicarius throws some light on the problems theologians were confronted with when they attempted to interpret their own times as Sacred History.

Hussitism - Miracle - Johannes Nider - Exempla - Bohemia 
